\title{
Patient Case Studies and Panel Discussion: Lymphoma
}

\section{ABSTRACT}

A heterogeneous group of diseases, lymphomas encompass a range of diagnoses that call for varied treatment approaches. Although some lymphomas require minimal intervention for cure or remission, others can be very difficult to treat and are associated with poor outcomes. At the NCCN 2019 Annual Congress: Hematologic Malignancies, a panel of experts used 3 case studies to develop an evidence-based approach for the treatment of patients with lymphomas. Moderated by Ranjana H. Advani, MD, the session focused on peripheral T-cell lymphoma, primary mediastinal large B-cell lymphoma, follicular lymphoma, and diffuse large B-cell lymphoma.

J Natl Compr Canc Netw 2019;17(11.5):1420-1423 doi: 10.6004/jncen.2019.5028

Lymphomas are a heterogeneous group of diseases with a range of diagnoses that require varied treatment approaches. Although some lymphomas require minimal intervention for cure or remission, others can be difficult to treat and are associated with poor patient outcomes. At the NCCN 2019 Annual Congress: Hematologic Malignancies, a panel of experts identified clinical challenges in managing patients with lymphoma. Moderated by Ranjana H. Advani, MD, Saul A. Rosenberg Professor of Lymphoma, Stanford Cancer Institute, the session focused on 3 case studies, which were used to develop an evidence-based approach for treatment of these patients. Panelists included Jeremy S. Abramson, MD, MMSc, Massachusetts General Hospital Cancer Center; Mrinal Dutia, MD, The Permanente Medical Group; Richard I. Fisher, MD, Fox Chase Cancer Center; and Andrew D. Zelenetz, MD, PhD, Memorial Sloan Kettering Cancer Center.

\section{Patient Case Study 1: Peripheral T-Cell Lymphoma}

In the first case study, a 44-year-old woman presented with a dry, nonproductive cough that she had been experiencing for 2 to 3 months, intermittent bouts of severe shortness of breath, decreased appetite, and a 20-pound weight loss. Chest radiograph revealed mild bilateral pleural effusion and bilateral pulmonary nodules, with the largest nodule measuring $5 \mathrm{~cm}$ in the right upper lobe. The patient was admitted for further evaluation, and additional testing was performed (see Figure 1 for results). Bone marrow evaluation revealed no morphologic abnormalities, but complex cytogenetics and the same T-cell clone was found as in the lung biopsy. Therefore, a diagnosis was made of stage IVB peripheral T-cell lymphoma (PTCL) with T-follicular helper (TFH) phenotype most consistent with angioimmunoblastic T-cell lymphoma (AITL).

Dr. Advani explained that this "complicated terminology" comes from recent updates to the WHO classification for PTCL. ${ }^{1,2}$ Furthermore, she explained that in recent years, advances in molecular biology have helped elucidate the underlying genetic complexity of PTCL and identify mutations and signaling pathways involved in lymphomagenesis. Importantly, many of the same genetic changes observed in AITL are also seen in PTCL not otherwise specified (NOS) that manifest a TFH phenotype. For this designation, the neoplastic cells should express at least 2 or 3 TFH-related antigens, including PD-1, CD10, BCL6, CXCL13, ICOS, SAP, and CCR5.

One potential treatment regimen to use in this patient population is CHOP (cyclophosphamide/doxorubicin/ vincristine/prednisone), which is associated with complete response (CR) rates of $50 \%$ to $60 \%$ in non-anaplastic large cell lymphoma (ALCL) and a 5-year overall survival rate of $40 \%$ to $60 \%$. According to Dr. Advani, patients with low International Prognostic Index (IPI) scores perform significantly better; however, relapse is associated with poor patient outcomes. "The ongoing challenge is to achieve higher CR rates and to translate those remissions into long-term survival," said Dr. Advani.

Another treatment option is the CHOEP regimen, which adds etoposide to CHOP. Retrospective studies suggest that outcomes may be better than historical data with $\mathrm{CHOP}^{3-5}$ In a prospective trial, the Nordic group evaluated the role of autologous stem cell transplantation after patients achieved a CR/partial response with $\mathrm{CHOEP}{ }^{6}$ At a median follow-up of 5 years, the 5-year progressionfree survival (PFS) and overall survival (OS) rates for AITL 


\section{$\mathrm{PET} / \mathrm{CT}$}

- Multiple FDG-avid liver lesions $\left(0.5-1.5 \mathrm{~cm}\right.$; SUV $\left.\mathrm{max}_{\max }, 5-8\right)$

- Mildly enlarged FDG-avid peridiaphragmatic prevascular lymph nodes $(1-2 \mathrm{~cm})$

- Diffuse ground glass and reticular pulmonary opacities with PET-avid bilateral pulmonary nodules RUL; largest nodule measuring $5 \times 3 \mathrm{~cm}$; SUV max 11

\section{VATS performed: RUL nodule}

- Polymorphous infiltrate composed of a mixture of

$>$ Small and large lymphocytes, plasma cells, eosinophils, and prominent high endothelial venules

$>$ Large atypical cells with prominent nucleoli resembling

Hodgkin Reed-Sternberg cells are seen

Immunohistochemistry: CD4+, CD30+ (scattered), CD10+, PD1+, BCL6+, CD56-, and ALK-; no loss of pan-T-cell antigens or expression of cytotoxic molecules

NGS studies: Positive for T-cell clone

Figure 1. Patient case study 1: results of further testing. Abbreviations: NGS, next-generation sequencing; RUL, right upper lobe; SUV, standard uptake value; VATS, video-assisted thoracic surgery.

were $49 \%$ and $52 \%$, respectively, and $38 \%$ and $47 \%$ for PTCL-NOS, respectively.

Finally, the phase III ECHELON-2 study was the first prospective trial in PTCL to show an OS benefit over CHOP. ${ }^{7}$ Frontline therapy for CD30-positive PTCL comparing brentuximab vedotin + cyclophosphamide/ doxorubicin/prednisone (BV-CHP) versus CHOP led to a significant improvement in PFS and OS for patients, with a comparable safety profile. The FDA subsequently approved brentuximab vedotin in combination with CHP for adults with previously untreated systematic ALCL or other CD30-expressing PTCL, including AITL and PTCL-NOS. "Based on these data, currently BV-CHP should be the standard regimen for untreated ALCL and other histologies that are CD30-postive," said Dr. Advani.

In the current case study, the patient received treatment with CHOEP and growth factor support. However, after 2 cycles, PET/CT scan showed no change in lung lesions with a worsening of right-sided pleural effusion (Deauville score 5). Thoracentesis of the pleural effusion was negative for infection but positive for abnormal T-cell population, with morphologic and immunophenotypic findings consistent with the initial diagnosis of PTCL with TFH phenotype most consistent with AITL. The patient was then started on single-agent brentuximab vedotin every 3 weeks. After 4 cycles, PET/CT scan revealed marked improvement in all lesions (Deauville score 4) and was continued on brentuximab vedotin for an additional 4 doses. PET/CT after 8 cycles revealed the resolution of lung nodules and adenopathy in the right axilla with increased metabolic activity (Deauville score 4).

Currently, 4 agents are FDA-approved for use in relapsed/refractory PTCL: pralatrexate, romidepsin, belinostat, and brentuximab vedotin. Aside from brentuximab vedotin use in ALCL, however, overall response rates are low. Brentuximab vedotin has been evaluated in relapsed/refractory AITL, showing an ORR of $54 \% .^{8}$ Responses did not correlate with level of CD30 expression.

Although no randomized studies have analyzed the role of consolidative hematopoietic stem cell transplantation, data from the prospective COMPLETE study suggested improved outcomes in patients with PTCL AITL and PTCL-NOS. ${ }^{9}$ On the other hand, said Dr. Advani, data from the LYSA study do not support the use of autologous stem cell transplant for upfront consolidation in patients with PTCL-NOS, AITL, or ALK-ALCL who have achieved a complete or partial response after induction. ${ }^{10}$

"If patients are young and have achieved remission or even a partial response, most of us tend to transplant because outcomes with relapsed disease are very poor," Dr. Advani concluded. "None of the approved drugs are home runs. The best chance you get is your first chance."

\section{Patient Case Study 2: Primary Mediastinal Large B-Cell Lymphoma}

In the second case study, a 21-year-old woman presented with severe cough and weight loss. Physical examination showed dilated veins on the anterior chest wall and no palpable peripheral lymphadenopathy. Chest radiograph revealed right-sided pleural effusion and a large mediastinal mass measuring $10.8 \mathrm{~cm}$. Additional testing was performed. PET/CT scan showed a bulky anterior mediastinal mass with $\mathrm{SUV}_{\max }$ of 24.1 and subcarinal and right hilar adenopathy. However, no evidence of disease was observed below the diaphragm. Bone marrow biopsy was negative. Mediastinoscopy with a biopsy of the mass showed diffuse lymphoid proliferation of intermediate-size atypical cells positive for CD20, CD79A, PAX5, CD30 (dim), MUM1, BCL2, and BCL6, and negative for CD10, BCL1, and EBER. Florescence in situ hybridization was negative for $M Y C$, $B C L 2$, and BCL6 rearrangements. The final diagnosis of primary mediastinal large B-cell lymphoma (PMBL) was made.

According to Dr. Advani, current treatment choices for PMBL are CHOP + rituximab (R-CHOP) with radiotherapy versus more intensive regimens, such as dose-adjusted etoposide/prednisone/vincristine/ cyclophosphamide/doxorubicin + rituximab (DA-EPOCH-R), without radiotherapy. In one study of DA-EPOCH-R 
with median follow-up of 8.4 years, PFS and OS were both $90 \% .{ }^{11}$ Another retrospective multicenter analysis that stratified patients by frontline regimen of either DA-EPOCH-R or R-CHOP showed a higher complete response with DA-EPOCH-R ( $84 \%$ vs $70 \%$; $P=.046$ ), but the patients in this arm were also more likely to experience treatment-related toxicities. ${ }^{12}$ At 2 years, $89 \%$ of patients in the R-CHOP arm and $91 \%$ of those in DA-EPOCH-R arm were still alive. Despite these similar outcomes, the consensus among panelists was that DA-EPOCH-R was the preferred option.

The patient in the current case report was started on DA-EPOCH-R, with an initial favorable response: interim imaging showed a reduction in size of the anterior mediastinal mass $(5 \times 6.3 \times 9.6 \mathrm{~cm})$ and resolution of left upper pulmonary nodules. The patient continued on DAEPOCH-R for 4 additional cycles. PET/CT scans 4 weeks posttreatment revealed a metabolically inactive, irregularly shaped anterior mediastinal mass $(5.5 \times 4 \times 5.2 \mathrm{~cm})$ and a focal area of low metabolic activity within the mass $\left(\mathrm{SUV}_{\max } 3.5\right.$; Deauville score 4$)$. She was asymptomatic, and the decision was made to follow with observation. After 2 months, PET/CT scans showed a stable mediastinal mass with increased metabolic activity $\left(\mathrm{SUV}_{\max } 6\right)$. Biopsy of the mediastinal mass was performed and immunohistochemistry results were consistent with relapsed PMBL; immunohistochemistry showed $30 \%$ of large cells were CD20- and CD30-positive.

"With PMBL, most of the cures are from initial therapy," said Dr. Advani. "Salvage rates in recurrent/ refractory disease are quite poor." Overall response rates in PMBL are $25 \%$ versus $48 \%$ for diffuse large B-cell lymphoma (DLBCL), and 2-year overall survival is only $15 \%$ versus $24 \%$, respectively. ${ }^{13}$

Although CD19 CAR T-cell therapy has been approved, said Dr. Advani, there are very limited data on its use in relapsed/refractory PMBL. According to Jeremy S. Abramson, MD, MMSc, Associate Professor of Medicine, Harvard Medical School, and Director of the Lymphoma Center, Massachusetts General Hospital Cancer Center, the limited available data are indeed encouraging in relapsed/refractory PMBL, and are "looking similar" to those for third-line treatment in DLBCL. Currently 3 randomized clinical trials are evaluating CAR T-cells versus autologous stem cell transplant (the current standard of care) as second-line therapy for relapsed/ refractory DLBCL, PMBL, and high-grade B-cell lymphomas, and these trials may ultimately change the standard of care in these patients.

Another treatment option for use in patients with relapsed/refractory PMBL is immune checkpoint inhibitors. Pembrolizumab has shown response rates of
$40 \%$ to $50 \%$ and is now approved by the FDA for use in the third-line and beyond. ${ }^{14}$ Furthermore, combination brentuximab vedotin + nivolumab showed even more robust activity, with an overall response rate of $73 \% .^{15}$

The patient in this case study received 5 cycles of pembrolizumab, and PET/CT scans showed a complete metabolic response.

\section{Patient Case Study 3: From Follicular Lymphoma to DLBCL}

In the last case study, a 48-year-old man presented with enlarged lymph nodes in his left neck and right groin measuring up to $2 \mathrm{~cm}$. He was asymptomatic and had no evidence of B symptoms. Laboratory test results were all normal. Excisional biopsy of the left cervical node showed follicular lymphoma (grade 1-2). PET/CT scan revealed left cervical, axillary, and right external iliac and inguinal adenopathy, with the largest node measuring $1.0 \times 2.0 \mathrm{~cm}\left(\mathrm{SUV}_{\max } 5.5\right)$. There was no splenomegaly or effusions. Bone marrow biopsy showed normal trilineage hematopoiesis. The diagnosis of stage IIIA follicular lymphoma (grade 1-2) was made, with a Follicular Lymphoma International Prognostic Index (FLIPI) score of 1 . Panelists agreed that the best approach for this patient was watchful waiting. Therapy was deferred, and the patient was followed with observation.

Two years later, the patient presented with enlarging lymph nodes in his left neck. He was anxious and had mild fatigue. Results of laboratory tests were normal. PET/CT scan showed increased adenopathy above and below the diaphragm, with the largest node (right external iliac node) measuring $3.4 \times 2.8 \mathrm{~cm}$ $\left(S_{U V} V_{\max }\right.$ 8.4). A biopsy of the right external iliac node was performed, with results showing follicular lymphoma (grade 1-2).

Panelists agreed that observation was an acceptable approach for an asymptomatic patients diagnosed with follicular lymphoma with low-volume disease. However, the patient opted for 4 doses of weekly rituximab. Repeat PET/CT (3 months posttreatment with rituximab) showed that most lymph nodes had resolved. Three years later, the patients presented with a new severe pain in the low back radiating down his right leg. MRI of the L-spine showed a T1 hypointense infiltrative mass replacing the L3 vertebral body. A core biopsy of right psoas mass showed that his follicular lymphoma had transformed to DLBCL, germinal center B-cell-like subtype, with double expressors of MYC $>40 \%$ and BCL2 $>50 \%$ and MYC translocation-negative, and expression of Ki67 was $90 \%$. IPI score was 4.

Andrew D. Zelenetz, MD, PhD, Professor of Medicine, Memorial Sloan Kettering Cancer Center, explained the 
unique biology of the "double-expressor" phenotype in DLBCL. "This is the one circumstance where DA-EPOCH-R may have a distinct benefit," he said. "However, in the absence of MYC translocation, it is not clear that overexpression of MYC, which is biologically driven without the translocation, shows a benefit from this intensive regimen."

The patient received 6 cycles of DA-EPOCH-R with 4 doses of intrathecal methotrexate. Results of interim and end-of-therapy PET scans showed a metabolic complete response (Deauville score 2).

Double-hit and double-expressor lymphomas tend to have inferior outcomes with R-CHOP therapy, Dr. Advani stated. Several retrospective studies and one prospective trial suggest improved outcomes with intensive chemotherapy for double-hit lymphomas. ${ }^{16-18}$ "Patients with a very high IPI, advanced-stage disease, or extranodal involvement are the ones that I consider good candidates for DA-EPOCH-R," she said, and also noted that doubleexpressor DLBCLs have a higher risk of central nervous system (CNS) relapse independent of CNS-IPI.

The patient in the current case study had a CNS-IPI of 3 and an $11 \%$ cumulative incidence of CNS relapse at 2 years. Data support some form of CNS prophylaxis, ${ }^{19}$ Dr. Advani concluded, but the jury is still out regarding optimal treatment for double-expressor non-Hodgkin's lymphoma.

Disclosures: Dr. Advani has disclosed that she has received grant/research support from Agensys, Inc., Celgene Corporation, Forty Seven, Inc. Janssen Pharmaceutica Products, LP, Kura Oncology, Inc., Merck \& Co. Inc., Millennium Pharmaceuticals, Inc., Genentech, Inc./Roche Laboratories, Inc., Pharmacyclics, Regeneron Pharmaceuticals, Inc. Seattle Genetics, Inc.; received consulting fees from AstraZeneca Pharmaceuticals LP, Bayer HealthCare, Gilead Sciences, Inc., Kite Pharma, Kyowa Hakko Kirin Co., Ltd., Cell Medica, Genentech, Inc./Roche Laboratories, Inc., Seattle Genetics, Inc., and Takeda Pharmaceuticals North America, Inc.; and is a scientific advisor for AstraZeneca Pharmaceuticals LP, Bayer HealthCare, Gilead Sciences, Inc. Kite Pharma, Kyowa Hakko Kirin Co., Ltd., Cell Medica, Genentech, Inc./Roche Laboratories, Inc., Seattle Genetics, Inc., and Takeda Pharmaceuticals North America, Inc. Dr. Abramson has disclosed that he receives consulting fees from AbbVie, Inc., Bayer HealthCare, Celgene Corporation, EMD Serono, Genentech, Inc., Janssen Pharmaceutica Products, LP, Karyopharm Therapeutics, Kite Pharma, and Roche Laboratories, Inc. Dr. Dutia has disclosed that she has no interests, arrangements, affiliations, or commercial interests with the manufacturers of any products discussed in this article or their competitors. Dr. Fisher has disclosed that he receives consulting fees from Celgene Corporation and PRIME. Dr. Zelenetz has disclosed that he received consulting fees from AbbVie, Inc., Amgen Inc., AstraZeneca Pharmaceuticals LP, Celgene Corporation, Gilead Sciences, Inc. Janssen Pharmaceutica Products, LP, Novartis Pharmaceuticals Corporation, Adaptive Biotechnologies Corporation, Genentech, Inc./Roche Laboratories, Inc., and Pharmacyclics; is a scientific advisor for AbbVie, Inc., AstraZeneca Pharmaceuticals LP, and MorphoSys AG; and receives grant/research support from BeiGene, Gilead Sciences, Inc. MEI Pharma Inc., and Roche Laboratories, Inc.

\section{References}

1. Swerdlow SH, Campo E, Pileri SA, et al. The 2016 revision of the World Health Organization classification of lymphoid neoplasms. Blood 2016; 127:2375-2390

2. Hildyard C, Shiekh S, Browning J, Collins GP. Toward a biology-driven treatment strategy for peripheral T-cell lymphoma. Clin Med Insights Blood Disord 2017;10:1179545X17705863.

3. Cederleuf $\mathrm{H}$, Bjerregard Pedersen $\mathrm{M}$, Jerkeman $\mathrm{M}$. The addition of etoposide to CHOP is associated with improved outcome in ALK+ adult anaplastic large cell lymphoma: a Nordic Lymphoma Group study. Br J Haematol 2017;178:739-746.

4. Schmitz N, Trümper L, Ziepert $M$, et al. Treatment and prognosis of mature T-cell and NK-cell lymphoma: an analysis of patients with T-cell lymphoma treated in studies of the German High-Grade Non-Hodgkin Lymphoma Study Group. Blood 2010;116:3418-3425.

5. Janikova A, Chloupkova R, Campr V, et al. First-line therapy for T cell lymphomas: a retrospective population-based analysis of $906 \mathrm{~T}$ cell lymphoma patients. Ann Hematol 2019;98:1961-1972.

6. d'Amore F, Relander T, Lauritzsen GF, et al. Up-front autologous stem-cell transplantation in peripheral T-cell lymphoma: NLG-T-01. J Clin Oncol 2012;30:3093-3099.

7. Horwitz $\mathrm{S}, \mathrm{O}^{\prime}$ Connor OA, Pro $\mathrm{B}$, et al. Brentuximab vedotin with chemotherapy for CD30-positive peripheral T-cell lymphoma (ECHELON-2): a global, double-blind, randomised, phase 3 trial. Lancet 2019;393: 229-240.

8. Horwitz SM, Advani RH, Bartlett NL, et al. Objective responses in relapsed T-cell lymphomas with single-agent brentuximab vedotin. Blood 2014; 123:3095-3100.

9. Park SI, Horwitz SM, Foss FM, et al. The role of autologous stem cell transplantation in patients with nodal peripheral T-cell lymphomas in first complete remission: Report from COMPLETE, a prospective, multicenter cohort study. Cancer 2019;125:1507-1517.

10. Fossard G, Broussais F, Coelho I, et al. Role of up-front autologous stem-cell transplantation in peripheral T-cell lymphoma for patients in response after induction: an analysis of patients from LYSA centers. Ann Oncol 2018;29:715-723.

11. Melani $C$, Advani R, Roschewski M et al. End-of-treatment and serial PET imaging in primary mediastinal B-cell lymphoma following dose-adjusted EPOCH-R: a paradigm shift in clinical decision making Haematologica 2018;103:1337-1344.

12. Shah NN, Szabo, Huntington SF, et al. R-CHOP versus dose-adjusted $\mathrm{R}-\mathrm{EPOCH}$ in frontline management of primary mediastinal B-cell lymphoma: a multi-centre analysis. Br J Haematol 2018;180:534-544.

13. Kuruvilla J, Pintilie M, Tsang R, et al. Salvage chemotherapy and autologous stem cell transplantation are inferior for relapsed or refractory primary mediastinal large B-cell lymphoma compared with diffuse large B-cell lymphoma. Leuk Lymphoma 2008;49:1329-1336.

14. Zinzani PL, Ribrag $\mathrm{V}$, Moskowitz $\mathrm{CH}$, et al. Safety and tolerability of pembrolizumab in patients with relapsed/refractory primary mediastinal large B-cell lymphoma. Blood 2017;130:267-270.

15. Zinzani PL, Santoro A, Gritt G, et al. Nivolumab combined with brentuximab vedotin for relapsed/refractory primary mediastinal large B-cell lymphoma: efficacy and safety from the phase II CheckMate 436 study [published online August 9, 2019]. J Clin Oncol. doi: 10.1200/ JCO.19.01492

16. Petrich AM, Gandhi M, Jovanovic B, et al. Impact of induction regimen and stem cell transplantation on outcomes in double-hit lymphoma: a multicenter retrospective analysis. Blood 2014;124:2354-2361.

17. Oki Y, Noorani M, Lin P, et al. Double hit lymphoma: the MD Anderson Cancer Center clinical experience. Br J Haematol 2014;166:891-901.

18. Dunleavy K, Fanale MA, Abramson JS, et al. Dose-adjusted EPOCH-R (etoposide, prednisone, vincristine, cyclophosphamide, doxorubicin, and rituximab) in untreated aggressive diffuse large B-cell lymphoma with MYC rearrangement: a prospective, multicentre, single-arm phase 2 study. Lancet Haematol 2018;5:e609-617.

19. Savage KJ, Slack GW, Mottok A, et al. Impact of dual expression of MYC and BCL2 by immunohistochemistry on the risk of CNS relapse in DLBCL. Blood 2016;127:2182-2188. 